\title{
Dossier Évolution et créationnisme La théorie de l'évolution a-t-elle une structure?
}

\author{
Olivier Perru \\ Histoire et philosophie des sciences, Université Lyon 1, EA4148, LEPS-LIRDHIST, 69622 Villeurbanne cedex, France
}

\begin{abstract}
Pour celui qui cherche à se plonger dans l'histoire des sciences de l'évolution, O. Perru propose de suivre le fil qui mène de L'Origine des espèces de Darwin à La Structure de la théorie de l'évolution de S.J. Gould. Le chemin à parcourir va de la théorie de la sélection naturelle du premier à celle de la sélection hiérarchique et des équilibres ponctués du second. Un tissage subtil apparaît : il associe les apports des biologistes et des paléontologues aux transformations des contextes philosophiques. Au bout du chemin la théorie de Gould s'avère à la fois intégrative et restrictive. On note au passage comment elle est utilisée dans la lecture idéologique et la technique éducative développées par le créationnisme actuel. $\mathrm{O}$. Perru fait enfin part de sa propre sensibilité. Il s'interroge : la théorie de l'évolution a-t-elle vraiment une structure? Il s'inquiète : faut-il se faire à l'idée d'une évolution par équilibres ponctués de l'espèce humaine?
\end{abstract}

La rédaction

\section{Introduction}

Stephen Jay Gould, l'initiateur américain de la théorie ponctualiste de l'évolution, est décédé en 2002. L'ouvrage auquel nous nous référons dans la présente étude critique est une traduction française, parue en 2006, de The Structure of Evolutionary Theory ${ }^{1}$, le dernier ouvrage de Gould, qui comprend plus de 2000 pages. L'auteur y annonce son intention de réviser la structure de la théorie de l'évolution et, donc, de réviser les caractéristiques de la logique darwinienne. Gould pratique une critique historique sans complaisance des apports de Darwin et de la théorie darwinienne ainsi que de «la synthèse moderne en tant que consensus restreint », dont il considère qu'elle est un durcissement de la théorie (p. 697). En deuxième partie de l'ouvrage, l'auteur propose la révision et l'élargissement de la théorie de l'évolution. Cet élargissement implique une enquête historique sur la "théorie de la sélection hiérarchique » (p. 898) et ses fondements, mais aussi l'apport de la proposition spécifique de Gould, l'équilibre ponctué. Le débat sur l'hypothèse de l'équilibre ponctué en termes d'alternative dans la théorie de l'évolution est particulièrement intéressant, bien que l'équilibre ponctué

Auteur correspondant : olivier.perru@univ-lyon1.fr

1 Gould, S.J., 2002. The Structure of Evolutionary Theory, Cambridge (Mass.), Harvard University Press. Trad. fr. : La Structure de la théorie de l'évolution, Paris, Gallimard, 2006. ne permette pas de prédire ou de modéliser n'importe quel phénomène évolutif. La fin de la seconde partie examine longuement le rapport dialectique entre contrainte et adaptation, afin de rendre compte de l'histoire évolutive du développement et de l'apparition de formes particulières à partir du potentiel propre aux structures organiques (exaptation, macroévolution, cf. p. 1648 et p. 1 697). La perspective fondamentale de Gould s'avère être une mise en synergie des phénomènes microévolutifs et macroévolutifs. Ce n'est ni une simple réfutation, ni une extension du darwinisme, mais une reconstruction de la structure et de la théorie de l'évolution autour « des relations supposées entre ontogenèse et phylogenèse » (p. 84).

Ces relations entre ontogenèse et phylogenèse permettent théoriquement d'envisager la transformation des organes et des espèces à travers l'histoire évolutive. Gould n'est pas très clair, à ce propos, en voulant sélectionner des « détails illustratifs bien choisis » pour réélaborer une structure théorique. Au commencement de ce procédé de révision, est annoncée plus clairement l'intention de tenir compte des macroévolutions et des extinctions de masse des espèces. Si l'auteur sélectionne des détails illustratifs et reprend de façon très documentée l'histoire des sciences de l'évolution, afin d'étayer les modèles évolutifs qu'il va proposer, il n'empêche que le noyau dur de cette théorie, posé au point de départ, englobe l'équilibre des 
micro- et macromutations au long des temps évolutifs et l'extinction/apparition en masse des espèces à une période donnée. C'est une hypothèse novatrice, qui a ses partisans et ses détracteurs dans les pays anglo-saxons comme dans les pays francophones. Il convient donc de l'analyser sous un angle à la fois positif et critique. Gould a beau vouloir faire œuvre de novateur et se défendre de construire son argumentation sur une simple réfutation ou correction du darwinisme, il n'empêche que la critique de Darwin est omniprésente dans cet ouvrage qui vise à infirmer le gradualisme en proposant l'idée d'équilibre entre les espèces, équilibre ponctué au cours des temps évolutifs par le rôle jugé fondamental des macromutations. L'enseignement qu'en tire l'épistémologue est une nouvelle approche des temps de l'évolution, approche qui semble réintégrer le catastrophisme pour rendre compte des «extinctions catastrophiques de masse » sans pour autant donner «un modèle exagérément simplifié de macroévolution » (p. 133). Par rapport à la théorie darwinienne classique, Gould donne une vision dialectique du temps, vision articulée sur la continuité et les ruptures. Le profane peut avoir l'impression que les mécanismes invoqués justifient peu l'immensité des temps évolutifs. Pourquoi de si longues «stases »? Pénétrons plus avant dans la cathédrale de Gould.

\section{Gould et l'histoire des sciences}

En fait, non seulement Gould fait l'inventaire de nombreux faits biologiques supposés démontrer les modèles et la théorie qu'il envisage, mais il donne aussi toute une enquête historique à travers les textes scientifiques des $\mathrm{XIX}^{\mathrm{e}}$ et $\mathrm{XX} \mathrm{X}^{\mathrm{e}}$ siècles. La dimension d'épistémologie et d'histoire des sciences est donc très présente dans cet ouvrage. De façon extrêmement significative, Gould présente l'influence de la Théologie naturelle (1802) de William Paley sur le point de vue de Darwin. «Paley a soutenu que Dieu manifeste son pouvoir créateur par l'exquise conformation des organismes à leurs fonctions immédiates ", c'est-à-dire, finalement, par leur adaptation (p. 171). En réalité, la «théologie naturelle » de Paley relève plus de l'apologétique que de la théologie. Celui-ci considérait, selon un point de vue répandu au XIX ${ }^{\mathrm{e}}$ siècle, que, si les organismes vivants sont beaucoup plus complexes dans leur formation et leur agencement que ne l'est une montre ou n'importe quel produit de l'art humain, c'est qu'ils "ont donc nécessairement été façonnés par une intelligence encore plus grande » (ibid.). En réalité, ce que Gould ne met pas bien en valeur, c'est que Darwin fait exactement du système de Paley ce qu'a fait Lamarck avec la «philosophie naturelle » du XVIII" siècle ou, pourraiton dire, ce qu'ont fait les posthégéliens avec l'œuvre de Hegel : il y supprime l'essentiel, c'est-à-dire Dieu, en le remplaçant par l'évolution et en conservant l'architecture du système, qui devient, du même coup, autonome. C'est le sort fatal des théologies naturelles du XVIII ${ }^{\mathrm{e}}$ siècle, qui avaient en fait oublié la métaphysique et qui reposaient beaucoup plus sur la foi que sur un fondement ontologique. Voir d'emblée Dieu à l'origine de l'ordre du monde, de la complexité et de la finalité qu'on y découvre, cela relève de la foi et non pas d'une métaphysique qui, elle, supposerait une progression intelligente dans les interrogations philosophiques, les jugements posés et les types de raisonnements inductifs ou a posteriori que le philosophe peut faire à partir de ce qui existe.

Darwin sécularise et inverse la théologie de Paley. La complexité et l'agencement des organismes se trouvent être le fruit de l'évolution, donc du hasard, de forces impersonnelles; l'ordre du monde n'a plus de sens et l'adaptation que Darwin retient de la théorie de Paley n'a plus rien à voir avec une activité créatrice. Elle correspond à une conséquence de la sélection naturelle et de la lutte pour la vie qui retiennent les individus les plus adaptés. On aboutit à l'exact contraire de la pensée de Paley, à partir pourtant d'une même mise en valeur de l'adaptation comme caractéristique des organismes. Dans le chapitre II de la première partie, Gould décrit assez longuement le rôle de la sélection naturelle, celui de la variation et le rythme du changement évolutif ainsi que son caractère continu. C'est une présentation sobre et systématique $\mathrm{du}$ gradualisme darwinien en tant qu'accumulation non orientée de changements de faible ampleur. C'est sur ces bases que Darwin a fondé son programme adaptationniste. Il est intéressant que Gould envisage les théories de l'évolution - la théorie darwinienne, la théorie synthétique et la sienne propre - en tant qu'elles impliquent un noyau fondamental et des conséquences, des modèles qui expriment et défendent ce noyau. Gould écrit à ce sujet (pp. 228-229) :

«On peut comprendre la thèse cruciale de Darwin sur la créativité de la sélection naturelle (et les notions en découlant : gradualisme, adaptationnisme et isotropie des variations) comme un système ayant prioritairement pour objectif de défendre cette façon vénérable et puissamment rationnelle d'envisager la nature et le changement [...]. Je suis convaincu que ces dernières [les critiques actuelles du darwinisme], considérées toutes ensemble, vont orienter la théorie de l'évolution en direction d'une structure explicative plus riche, conservant cependant un noyau darwinien. »

En fait, ce sur quoi Gould fait porter sa critique du darwinisme, ce sont les catastrophes naturelles en tant qu'elles impliquent des phénomènes d'extinction de masse, interprétables en dehors du cadre darwinien. Plus la rapidité de certains changements serait responsable de caractéristiques du tableau évolutif, plus celui-ci échapperait à la vision darwinienne générale. Tel est le point de vue de Gould, mais peut-on vraiment opposer catastrophisme et darwinisme? Les épisodes d'extinction de masse, dus à des catastrophes naturelles, ne constitueraient-ils pas 
qu'une parenthèse dans un processus d'évolution globalement darwinien? De ce point de vue, il est intéressant de voir le regard que porte Gould sur les théories qui ont précédé celle de Darwin et sur les autres formes de déterminisme évolutif avant et après Darwin.

Chez ce dernier, Gould distingue deux principes d'évolution : le principe de divergence des caractères à partir d'une forme ancestrale et le principe de sélection naturelle qui retient les formes les plus adaptées à telle circonstance de «l'économie de la nature » (p. 320). De fait, ce qui est plus fondamental dans le système de Darwin est ce qu'on appelle aujourd'hui la variabilité génétique. La divergence des caractères telle qu'elle existe dans la nature serait le résultat de la sélection naturelle, laquelle « favorise généralement, au sein du spectre des variants issus d'une souche parentale donnée, les formes les plus divergentes, les plus différentes, les plus extrêmes » (p. 334). Gould fait remarquer, dans la suite de son développement, que la sélection darwinienne porte d'abord sur les organismes et qu'il aurait échoué dans sa recherche d'explication de la diversité des espèces. Ce qui pose toujours problème est le rapport entre l'adaptation des variants individuels à un milieu et la création d'une nouvelle espèce. Le succès individuel n'explique donc pas la transformation en une nouvelle espèce. Il est vrai que Darwin manque d'un mécanisme explicatif pour passer du niveau de la sélection naturelle des " organismes variants extrêmes » à celui de « la multiplication des espèces » (nouvelles); ce mécanisme (la mutation) ne sera acquis qu'avec la théorie synthétique de l'évolution, dans la première partie du XXe siècle (p. 340).

Dans cette revue de l'histoire de la biologie transformiste au XIX ${ }^{\mathrm{e}}$ siècle que fait Gould, une part importante de la réflexion est consacrée aux "déterminismes internes ", c'est-à-dire à l'évolution morphologique et structurale, illustrée entre autres par Geoffroy Saint-Hilaire ou Owen. En réalité, Gould estime que le projet darwinien fut un échec en son temps. Il ne pense pas en termes d'exclusivisme : s'il s'agit de rejeter l'exclusivisme de l'adaptation darwinienne, telle qu'elle apparaît au terme de la théorie synthétique, il s'agit aussi de penser la biologie évolutive en termes de complémentarité (entre les caractères propres à l'émergence des espèces et l'adaptation darwinienne, par exemple). Ainsi, l'auteur consacre quelques dizaines de pages (ch. IV, p. 355-474) aux déterminismes internes et aux lois de la forme comme alternatives au fonctionnalisme de Darwin et de l'école anglaise. La carence de la théorie de Darwin est qu'il aurait expliqué de façon satisfaisante, par l'adaptation, le succès différentiel des organismes mais pas celui des espèces, d'où la nécessité de relier l'apparition et l'extinction des espèces à la notion de contraintes structurales.

Dans L'Origine des espèces, Darwin imagine un ensemble de mécanismes déterminant l'extinction de certaines espèces, plus vulnérables que les autres, du point de vue du nombre ou de l'adaptation. Or, Gould considère que le raisonnement darwinien sur le rapport entre « la sélection des variants extrêmes » et « la diversification » ne tient pas (p. 351), que son schéma fonctionnaliste uniforme ne peut expliquer ni la diversification ni l'extinction des espèces. "L'élimination des formes ancestrales par la sélection naturelle, dans le cadre d'une compétition avec leurs descendants ", est incompréhensible dans L'Origine des espèces (ibid.). Selon Gould, le choix fondamental de Darwin sur l'apparition des structures ancestrales communes est celui de «l'action de la sélection naturelle en tant qu'adaptations aux conditions organiques et inorganiques de l'existence dans des milieux ancestraux » (p. 359). Il souligne que, selon Darwin, l'unité de type chez les animaux d'une même classe et l'adaptation aux conditions d'existence sont tributaires ensemble de la sélection naturelle.

Il est très important ici de bien mettre en valeur ce que l'on pourrait considérer comme la réponse de Gould à Darwin. En fait, l'auteur a bien vu le talon d'Achille de la position darwinienne : le rôle des contraintes internes de structure et de forme disparaît pratiquement du champ de l'évolution. On comprend alors que, pour présenter les alternatives au darwinisme, Gould retrace l'histoire du structuralisme morphologique au XIX ${ }^{\mathrm{e}}$ siècle : la théologie créationniste de Louis Agassiz où l'ordre taxinomique révèle la nature et la création, le débat prédarwinien autour de l'unité de plan (autour de Geoffroy Saint-Hilaire et de Cuvier et autour du point de vue de Goethe, contre la primauté des explications adaptationnistes, $c f$. p. 406). Puis il expose «l'intérêt vif mais limité de Darwin pour les contraintes structurales» (p. 458). Darwin «se préoccupait sérieusement des contraintes structurales » (p. 462), qu'il reliait «aux mécanismes internes de la variation» (p. 463). Certes, il s'est intéressé aux variations corrélatives de forme chez les animaux, ces variations et ces contraintes internes de l'organisme étant subalternées à la sélection naturelle. Expliquer la variation était une difficulté réelle de Darwin, d'où l'intérêt pour les variations corrélatives et homologues. L'intuition de Gould est d'accorder plus d'importance que dans le darwinisme aux «formes revêtues par la variation », par la révision de la théorie darwinienne de la sélection naturelle.

Nous ne pouvons pas aborder tous les aspects historiques que convoque Gould dans son ouvrage. Il s'intéresse en particulier aux apports de Francis Galton concernant la régression à la moyenne. « Pour lui, l'existence de la régression signifiait que la variation continue ne pouvait conduire au changement évolutif progressif parce que toutes les variations extrêmes favorables allaient, au cours des générations successives, régresser vers la moyenne, et il ne pouvait donc en résulter aucune modification évolutive permanente ou orientée dans un sens donné » (p. 477). Galton conteste donc la nécessité de petites variations continues pour l'obtention du changement évolutif. Selon 
Gould, il considère au contraire qu'il faut qu'apparaisse une variation discontinue anormale tout en demeurant conforme au type ${ }^{2}$. On comprend alors ce qui intéresse Gould dans le point de vue de Galton, sur qui, plus que sur Darwin, il va fonder son propre point de vue : il s'agit de la «discontinuité comme base de la variation efficace» et de la «canalisation du changement dans des directions préférentielles en raison de facteurs internes (contraintes) » (p. 478). Des scientifiques tels que William Bateson ou Hugo De Vries se sont inspirés du point de vue de Galton. Le long commentaire que Gould consacre à la théorie de Bateson lui permet d'invoquer la saltation comme modalité de variation et de changement évolutif. Dans Materials for the Study of the Variation (1894) ${ }^{3}$, Bateson découvre l'homéose comme étant une variation génétique correspondant à des structures répétées ; ce travail est à l'origine des recherches modernes de génétique du développement jusqu'aujourd'hui. Fondamentalement et dans la logique de la zoologie du XIX ${ }^{\mathrm{e}}$ siècle, de Moquin-Tandon à Edmond Perrier, Bateson affirme que les segments sont « représentables comme des entités anatomiques autonomes » (p. 557). Chez les arthropodes, la différence du nombre de segments ou d'articles dans les antennes et les membres d'espèces voisines conduit à montrer que l'évolution ne peut pas être totalement graduelle, mais que l'acquisition d'un nouveau segment ou d'un nouvel article est un phénomène discontinu. Cette remise en cause du gradualisme s'accompagne d'un doute sur le rôle de la sélection naturelle : Bateson, et Gould à sa suite, s'interrogent sur le rôle de la sélection dans la production d'une espèce de blatte ayant un tarse à quatre articles par rapport à la variété «normale » à cinq articles. Il semble que Darwin ait répondu ici par l'affirmation de la relation essentielle entre l'environnement et l'évolution : il aurait cherché dans quelle mesure l'une et l'autre des variétés reflètent une adaptation à des conditions de milieu; au contraire, Gould considère que l'adaptation au milieu ne peut pas expliquer grand-chose de cette variation.

En réalité, Bateson cherchait à se fonder sur une démarche scientifique par expérimentation et démonstration. Tout son discours tend à montrer que Darwin n'explique pas l'origine de la variation, mais la sélection des variations favorables. De fait, dans le darwinisme, avant l'explication des variations par les mutations, c'est-à-dire avant la synthèse néodarwinienne de la sélection et des mutations, la démarche scientifique reste hypothétique, à partir de l'observable et corroborée par les observations

\footnotetext{
${ }^{2}$ C'est le modèle du polyèdre de Galton, dans lequel l'équilibre instable peut faire basculer à tout moment le polyèdre d'une face à l'autre. Ce modèle est utilisé pour symboliser le passage par un changement brusque d'une phase d'équilibre (instable) à une autre conformation, plus stable par rapport au milieu.

3 Bateson, W., 1894. Materials for the Study of the Variation, London, MacMillan.
}

de Darwin lui-même. Rappelons aussi que la recherche de Bateson sur les causes de l'hérédité et des variations fut, en son temps, un échec.

L'autre personnage qu'évoque Gould à propos des variations est Hugo De Vries. On sait que celui-ci a étudié pendant plus de 20 ans l'apparition de ce qu'il a considéré comme étant de nouvelles espèces d'œnothères, et qui n'étaient probablement que des individus manifestant les formes ancestrales à la suite de croisements successifs. De Vries s'intéresse à une explication de ces phénotypes par l'hérédité, qu'il situe au niveau de pangènes qui ne migrent plus à l'intérieur des cellules, comme chez Darwin, d'où une négation implicite de l'hérédité des caractères acquis. La théorie des mutations de De Vries prétend donc bien donner la cause d'une variation apparaissant soudainement, et Gould insiste sur cet antigradualisme supposé de De Vries. Il écrit : «Par-dessus tout, les mutants apparaissaient de façon soudaine et se transmettaient héréditairement en restant conformes au type » (p. 592). La mutation serait donc essentiellement discontinuité, saltation ; la soudaineté ${ }^{4}$ et l'accessibilité apparente de mutations démentiraient « les changements lents et graduels envisagés par Wallace et ses disciples » (p. 595) ${ }^{5}$. C'est en fait l'action de la sélection naturelle qui peut provoquer un changement graduel à partir des variations rares : c'est bien l'expansion de nouvelles espèces qui se fait graduellement, non leur apparition. Ainsi, le texte de De Vries lui-même affirme qu'il règne une harmonie entre la théorie de la sélection, les travaux de Mendel compris depuis peu à la lumière des découvertes de la cytologie et les apports de la théorie des mutations.

Un autre auteur qui soutenait l'apparition de nouvelles espèces par des mutations brusques, Richard Goldschmidt (cf. p. 627), tenta de faire la différence entre macroévolution et microévolution. Gould affirme que Goldschmidt distingue la microévolution, qui assure l'adaptation de l'organisme, de la macroévolution qui, elle, se situe non plus au niveau de l'évolution de l'organisme, mais à celui des espèces ( $c f$. p. 629). Cela rejoint la distinction qu'il a faite entre l'adaptation et la complexification productrice de nouveau (au sujet de Lamarck) ou la distinction

\footnotetext{
${ }^{4}$ Pour critiquable que soit le livre de De Vries, Die Mutationstheorie, Erster Band: Die Entstehung der Arten durch Mutation (Leipzig, Veit, 1901), il va complètement révolutionner l'approche de l'évolution au début du $X X^{\mathrm{e}}$ siècle. L'auteur retrace toutes les observations et les expérimentations qu'il a menées autour d'Amsterdam, depuis 1875 . Il remarque qu'ayant observé des caractères anormaux en plein champ, il en obtint très peu en culture. Il s'agirait simplement du retour aux caractères ancestraux suite à des croisements d'espèces hybrides. Ces croisements peuvent effectivement faire apparaître des caractères dominants, caractéristiques de l'espèce mère (type sauvage) ou des caractères récessifs correspondant à des mutations.

${ }^{5}$ D'après cette interprétation de Gould, De Vries aurait donc rejeté la responsabilité du gradualisme comme pensée scientifique sur Wallace et non sur Darwin.
} 
entre l'adaptation des meilleurs variants et la production efficace d'espèces nouvelles (au sujet de Darwin). Mais peut-on systématiser et sur quelles bases, à travers l'histoire des sciences, cette distinction de deux tendances évolutives? Il faudra réinterroger dans le contexte actuel cette affirmation de Gould. Après De Vries, historiquement, tous les éléments étaient réunis pour la synthèse néodarwinienne, Gould ne faisant ici que commenter les textes fondateurs de cette synthèse. Ronald Fisher réalisa le premier essai de synthèse en intégrant «l'hérédité particulière mendélienne » dans la vision évolutive d'une «variation continue et isotrope des phénotypes » (p. 701). De cette première phrase de la théorie synthétique, date un rejet définitif du lamarckisme, se basant sur l'attribution d'une explication mendélienne à « la variabilité continue et de petite dimension » (p. 707). C'est aussi la période de fondation de la génétique des populations.

L'auteur plaide ensuite pour un durcissement de la synthèse autour de Theodosius Dobzhansky. Dans les éditions successives de Genetics and the Origin of Species, Dobzhansky met de plus en plus en valeur le programme adaptationniste, insistant sur le «pouvoir de la sélection naturelle » et la «nature adaptative de la plupart des changements évolutifs » (p. 730). Cette tendance se révèle dans la notion de paysage adaptatif : Dobzhansky chercha une solution à la discontinuité phénotypique des grands groupes de vertébrés. Le paysage adaptatif est discontinu et se compose d'un ensemble de pics qui correspondent aux solutions optimales d'adaptation, les pics adjacents symbolisant l'adaptation d'un groupe zoologique. "La discontinuité dans l'espace taxinomique recouvre la discontinuité dans les formes correspondant optimalement aux environnements disponibles, l'adaptation étant l'agent assurant la distribution exacte des formes aux lieux qui leur conviennent » (p. 732). Et Gould conclut : «Ainsi Dobzhansky explique la structure hiérarchique de la classification par l'insertion appropriée des clades au sein des espaces écologiques préexistants » (p. 732). Si Gould met en valeur cette interprétation de Dobzhansky dans la dernière édition de Genetics and the Origin of Species, c'est pour la mieux contrecarrer : un groupe zoologique dérive d'un ancêtre commun et c'est ce qui explique leur morphologie fondamentale, même si cet ancêtre commun et ses descendants ont été bien adaptés à leur environnement. «Toutes les espèces de félidés actuelles ont hérité du Bauplan unique en son genre du chat, et n'ont pu s'écarter beaucoup de cette structure commune tandis qu'elles se sont adaptées à leur mode de vie, chacune à sa manière » (p. 733). Après Dobzhansky et Simpson, Gould cherche à montrer qu'Ernst Mayr serait aussi passé d'une position initiale pluraliste (dans un ouvrage publié en 1942 , où il mettait l'accent sur la discontinuité des espèces) à une position insistant (en 1963) sur une primauté de la sélection au niveau de petites populations de l'espèce souche $^{6}$. Mais y a-t-il vraiment un changement de perspective chez Mayr entre 1942 et 1963 ? Concernant tous ces auteurs, on peut critiquer chez Gould une part de volonté idéologique de souligner un retour de l'adapationnisme et du gradualisme, dans un durcissement de la synthèse. En réalité, les éléments adaptationnistes et gradualistes ont toujours été présents ; ce qu'il faut noter, c'est l'élaboration scientifique et mathématique croissante de la génétique des populations, qui permit une formalisation plus précise de l'adaptation vers 1960.

Certes, il est vrai que la synthèse moderne aboutit à une réduction des possibilités de macroévolution et à un triomphe du gradualisme, et c'est ce qu'on en retient généralement, en particulier du fait de la littérature de vulgarisation et des manuels scolaires. L'évolution a souvent été présentée comme le fruit de "tendances évolutives » où le phénomène de spéciation et la notion même d'espèce sont mis entre parenthèses ( $c f . p .781$ ). C'était le point de vue de Simpson. Un autre point souligné à raison par Gould est la vulgarisation (dans les manuels scolaires) de l'équilibre sélection-mutation, vu comme facteur premier de l'évolution.

\section{La théorie ponctualiste}

Rompant avec la synthèse moderne, Gould se base sur une notion individualiste de l'espèce. L'espèce en tant qu'entité d'évolution est-elle concevable comme individualité ? On pourrait se demander pourquoi cette question : la raison en est de distinguer les caractères essentiels de l'espèce dans le flux continu de l'évolution; l'enjeu est d'envisager l'évolution comme « un processus réalisant des scissions successives au niveau spécifique » et non comme une tendance graduelle et continue. Il y a donc une hypothèse sous-jacente à toute cette deuxième partie de l'ouvrage de Gould : c'est l'affirmation d'irréductibles discontinuités entre les espèces et leur conciliation avec le phénomène évolutif, et ceci, en vue d'amener l'hypothèse que l'on retient généralement comme première dans l'œuvre de Gould : la coexistence de longues périodes d'équilibre et de brusques changements, mouvements d'extinction et d'apparition d'espèces dans l'évolution biologique.

L'individualité de l'espèce pose, bien entendu, des problèmes redoutables. L'auteur note la réticence des biologistes à cet égard ( $c f$. p. 840$)$. En réalité, ce problème n'est pas posé par Gould du point de vue de la vie biologique des organismes et des populations d'une même espèce,

\footnotetext{
${ }^{6}$ Mayr, E., 1942. Change of genetic environment and evolution, in Huxley, J.S., Hardy, A.C., Ford, E.B. (Eds), Evolution as a Process, London, G. Allen and Unwin, 157-180 ; Mayr, E., 1963. Animal Species and Evolution, Cambridge (Mass.), Harvard University Press.
} 
mais du côté de la durée de l'espèce. L'espèce a une durée de vie repérable, avec des étapes, et cette vie assume à la fois la stabilité et un minimum de changements. Bien entendu, cet essai de définition ne fait pas de l'espèce ni de la population des êtres vivants. Mais l'espèce n'est pas au sens strict un individu, comme le pense Gould, elle est une information génétique qui s'individualise et se réalise dans une succession d'individuations physiques. L'espèce se réalise dans la multiplicité des organismes qui la constituent, son essence est l'information biologique qui circule entre ces organismes. Du point de vue de la durée des phénomènes de la vie, par contre, il est possible $\mathrm{d}$ 'individualiser l'espèce entre naissance et mort, dans l'évolution. Ce que Gould recherche est situé au-delà de l'individualité telle qu'on la saisit habituellement, c'est une individualité "au sens spécifiquement évolutionniste », c'est-à-dire prenant sens idéalement dans cette théorie et corroborée par des faits (p. 851). L'espèce dans la durée serait une « unité de base » de la «macroévolution », ce qui repose la question de l'action de la sélection. Pour relativiser l'action de la sélection sur l'individu, Gould remet en cause ce qu'il appelle le "sélectionnisme génique », c'est-à-dire une vision univoque de l'action prioritaire de la sélection sur les gènes, sans tenir compte des propriétés des organismes biologiques ni des groupes.

Gould considère donc les gènes comme des unités comptables du changement, parce que la fréquence des gènes modifiés comptabilise les changements évolutifs survenus. Il voit, dans l'action supposée de la sélection sur les gènes, une confusion entre compatibilité et causalité. En effet, «la sélection naturelle est un processus causal» (p. 881) et ce sont les organismes et les groupes, les populations qui subissent directement l'action de la sélection naturelle par les gènes. Il est cependant aisé de montrer que, si la sélection agit sur les organismes les mieux adaptés, elle agit indirectement sur la permanence des gènes qu'ils transmettent, à travers les générations. L'argumentation de Gould a donc quelque chose de sophistique; on peut admettre que les unités directes de sélection ne soient pas les gènes, même si ces derniers sont, in fine, les ultimes objets du processus de sélection par l'intermédiaire des individus, des espèces et des populations (ce que Gould admet d'ailleurs). Gould propose une théorie hiérarchique de la sélection, parce que, pour lui, les espèces sont les premières visées par la sélection naturelle, puis les populations et les organismes et enfin, au plus bas niveau dans la hiérarchie, les gènes, qui «possèdent la propriété unique en son genre d'enregistrer les changements » (p. 887). Gould réfute les arguments contre la sélection entre espèces, il fait remarquer que ces arguments sont généralement pertinents pour des dèmes (les dèmes des souris vivant dans des meules de foin, par exemple), mais qu'ils le sont beaucoup moins du point de vue de la sélection entre espèces. Par contre, dans certaines conditions, il existe un risque, «la sélection organismique surpasse nécessairement la sélection entre espèces et annule ses effets » (p. 908).

À ce moment, intervient un changement de registre extrêmement important pour comprendre le propos de l'auteur. La puissance de la sélection des organismes demeure dans le possible de la génétique moléculaire, mais «les archives observables de la grande majorité des espèces fossiles bien étudiées montrent qu'elles sont en stase tout au long des strates géologiques où elles figurent » (p. 909). La paléontologie est convoquée pour établir les stases et supporter l'hypothèse d'une sélection interspécifique révélant des tendances de l'évolution au sein des clades. Une difficulté demeure cependant : mieux définir la sélection entre espèces. La notion de caractère émergent semble décisive pour mieux cerner l'apparition d'espèces nouvelles; mais elle rend ardue la prise en compte des divers traits des populations ou de la variabilité. La notion de caractère émergent, si elle fait apparaître des cas concrets d'adaptation propres aux espèces, « réduit peut-être sans nécessité la sphère dans laquelle intervient la sélection entre espèces » (p. 924) et elle est tout simplement difficile à définir et à établir explicitement ${ }^{7}$. Nous rejoignons Gould sur le fait que la mise à l'épreuve de ces caractères est hasardeuse et qu'il est très difficile de distinguer un véritable caractère émergent au niveau de l'espèce et des effets secondaires d'un caractère situé à un niveau inférieur à l'espèce dans la hiérarchie évolutive.

$\mathrm{Au}$ terme d'une longue discussion, Gould en vient à considérer les espèces non seulement comme des individus, mais comme des interacteurs. La question est évidemment de savoir si les espèces qui vivent dans des environnements variés et ont une aire de répartition très vaste peuvent être des unités de sélection. Gould affirme que l'interaction des unités de sélection ne demande pas forcément qu'elles vivent en sympathie (cf. p. 987) ni qu'elles soient soumises à une compétition biotique; l'interaction entre espèces peut se limiter à une compétition abiotique, à une lutte pour la survie face aux facteurs environnementaux. Il considère comme efficace la sélection entre espèces dans leur ensemble plus que la sélection entre clades, sans toutefois exclure cette dernière dans des cas précis : les clades de mollusques à la fin du Crétacé, les dinosaures.

L'explication macroévolutionniste se base sur les espèces « en tant qu'unités stables et fondamentales du changement » (p. 1003) et suppose un changement unidirectionnel déterminant une tendance évolutive, un changement non aléatoire mais orienté, la sélection jouant a posteriori et entre espèces. "La spéciation unidirectionnelle, lorsqu'elle s'appuie sur des caractéristiques irréductibles du niveau de l'espèce, représente une modalité indépendante

\footnotetext{
${ }^{7}$ Les caractères non émergents influant sur l'adaptation par des effets secondaires sont des exaptations.
} 
de macroévolution qui est déterminée causalement et qui n'est pas fondée sur la sélection entre espèces » (p. 1015). On se demande toujours un peu ce qui fonde ce type d'affirmation chez Gould : ici, c'est l'existence d'un changement directionnel reproductif (pression de mutation). Gould introduit ainsi, et sans beaucoup d'exemples, la ponctuation : les espèces sont en état d'équilibre pendant de très longues périodes, mais il existe des points qui révèlent un changement sous l'impulsion de la sélection entre espèces. Une variation nouvelle est « adaptative au niveau de l'espèce » et la synergie entre « les intérêts des organismes et ceux des espèces est à l'origine d'une puissante accélération de la macroévolution » (p. 1039).

Les observations de paléontologie permettraient d'établir la ponctuation comme base d'une nouvelle théorie de l'évolution. À partir des observations (des mollusques d'eau douce des lacs africains, par exemple), la difficulté est de "définir des ponctuations par rapport à la durée de la stase des espèces qui en sont issues » (p. 1075), les espèces acquérant leurs caractères distinctifs au moment de la ponctuation. Gould évoque les travaux de Peter Williamson, qui a mis en évidence " un phénomène remarquable de changement de la variabilité au sein d'une population en cours de spéciation ", durant environ un millier d'années, parce qu'il a utilisé des sédiments des grands lacs africains où les vitesses de sédimentation sont très élevées (p. 1077). Sur l'évolution de l'espèce actuelle d'escargot Cerion rubicundum, Gould suggère une transition évolutive de 15000 à 20000 ans entre l'espèce ancestrale et l'espèce descendante. La théorie de l'équilibre ponctué est évidemment étayée par des données expérimentales; encore faut-il arriver à démontrer que les phénomènes de ponctuation ne sont pas simplement des cas singuliers, mais qu'ils sont dominants dans l'évolution. Là est vraiment l'originalité des thèses de Stephen Jay Gould. C'est l'idée d'un mécanisme dominant de spéciation, "suffisamment rapide dans le temps » et « localisé dans l'espace » (p. 1091). Reste ensuite à rentrer dans la discussion scientifique visant à reconnaître les espèces comme des individus évoluant par macroévolution, et avec le rythme évolutif de la ponctuation et de la stase. On aura compris que les confirmations de cette théorie générale de l'évolution sont à rechercher dans les données paléontologiques.

En réalité, on peut logiquement se demander si Gould n'est pas conduit à attribuer un rôle majeur à la macroévolution et aux ponctuations du fait même de la «nature des archives fossiles », qui sont souvent discontinues et livrent des épisodes distincts entourant la spéciation (p. 1106). Comme nous l'avons déjà supposé, la théorie des équilibres ponctués est évidemment tributaire de la spécialité de Gould, la paléontologie. La persistance de l'ancêtre lors de la constitution de l'espèce descendante est considérée par Gould, à juste titre, comme un signe sûr de l'existence de l'équilibre ponctué. Le problème demeure néanmoins de définir le degré d'universalité de ce phénomène. Étayer l'existence de ce phénomène par des observations dans un tel contexte est une chose, l'universalité de la théorie pose un problème tout autre. Gould le pressent et admet la probabilité de prédominance du gradualisme chez les foraminifères planctoniques, tout en réfutant l'usage de ce fait comme preuve universelle : il pourrait y avoir « une disparité fondamentale des modes d'évolution selon les taxa » (p. 1127). Si l'équilibre ponctué concernait en priorité les métazoaires, il faudrait aussi rappeler que cette théorie s'applique à l'évolution des phénotypes (et non des génotypes). L'observation de la succession des espèces sur les archives fossiles montre que la cladogenèse correspond aux équilibres ponctués et se traduit par « la production répétitive de plusieurs espèces descendantes à partir d'une espèce ancestrale restant invariable » (p. 1149). Dans cette logique, Gould plaide donc pour la prédominance de la cladogenèse sur l'anagenèse. Il passe en revue des exemples de stases et d'évolution brusque des échinodermes dans des coupes stratigraphiques et s'intéresse à l'allure générale des stases chez ces animaux, à la différence du gradualisme "sans doute authentique » des foraminifères (p. 1162). Les constatations amènent à l'interrogation sur les causes des ponctuations. L'apparition d'espèces nouvelles par cladogenèse semble répondre à des changements décisifs, géographiques et environnementaux. À la différence du gradualisme, le ponctualisme ne demande pas «la mise en œuvre de nombreuses causes pendant des durées importantes », mais résumerait les «multiples conséquences, liées entre elles, de la mise en jeu d'un seul facteur (ou d'un petit nombre de facteurs » (p. 1191). Plusieurs caractères peuvent être modifiés simultanément par un changement unique, c'est l'exemple déjà cité du mollusque Cerion étudié par Gould. Les environnements changeant de manière fluctuante sur de longues périodes provoquent des stases, alors que ce sont les environnements stables qui favorisent les adaptations graduelles.

Cette théorie des équilibres ponctués a donc des conséquences pratiques, en particulier à l'égard du darwinisme. Gould rappelle que « Liebermann et Dudgeon (1996) ont expliqué la stase en tant que réponse à la sélection naturelle de la part d'espèces subdivisées (comme la plupart le sont probablement) en populations... » (p. 1232). La stase correspond à une stabilité phénotypique qui serait une réponse à la sélection : comment concilier cette stabilité avec l'efficacité de la sélection naturelle sur l'adaptation des caractères (constatée depuis les célèbres observations de Darwin sur le bec des pinsons) ? Sans doute en considérant que ce sont des sous-populations qui s'adaptent graduellement, la totalité de l'espèce se révélant être un compromis, un type moyen stable entre plusieurs morphologies concurrentes. Cette argumentation antigradualiste n'est pas totalement convaincante. De fait, les populations de pinsons de Darwin évoluent sur de courtes périodes, 
selon les changements climatiques ; mais un changement climatique plus long et plus généralisé ne consacre-t-il pas la suprématie des individus plus adaptés par des modifications mineures de caractères?

À partir de l'homogénéité des données paléontologiques, dans la théorie ponctualiste, on en arrive finalement à l'existence de " ponctuations partout, du haut en bas de la hiérarchie de la nature » (p. 1290), et donc à une généralisation de l'extinction des espèces existantes et à la production de nouvelles espèces, dans un bref délai ${ }^{8}$. Il y a un point essentiel que Gould ne précise pas : cette théorie demande à se traduire dans des modèles qui ne sont pas des représentations du changement mais des formalisations causales. L'application du modèle du tas de sable de Per Bak, énoncé au début des années 1990, a été très utilisée pour faire comprendre les avalanches d'extinctions d'espèces. Un autre auteur, Stuart Kauffman, intervient dans le même sens : en partant de la coévolution, il montre l'apparition de nouvelles espèces comme le fruit d'interactions multipliées dans un système. La dynamique interne du système (non linéaire) explique donc aussi bien l'équilibre que le changement brusque. Ainsi, ces simulations semblent aller dans le sens de la théorie ponctualiste sans même qu'il y ait besoin de faire appel à des facteurs environnementaux externes au système. Des paléontologues comme Blackburn ont accumulé les données expérimentales en vue de démontrer la ségrégation ponctuationniste entre animaux ovipares et vivipares. Sur le plan embryologique, la placentation peut être vue comme un corrélat nécessaire de la viviparité. Sur le plan paléontologique, l'apparition récente et rapide des lignées vivipares est en faveur de la ponctuation. L'état intermédiaire, la «rétention des œufs » dans les voies génitales des femelles des espèces ovipares, serait peu durable (p. 1326). Les données paléontologiques montrent une forte ressemblance "entre espèces con-génériques ovipares et vivipares » (p. 1327). On aurait là un cadre paradigmatique de l'apparition de nombreuses espèces vivipares à des périodes souvent récentes (Pliocène, Pléistocène).

Un moment plus contestable de l'exposé de Gould est l'application des équilibres ponctués aux étapes récentes de l'évolution des hominidés et d'Homo sapiens. Il semble hasardeux d'imaginer un développement technologique, artistique et culturel d'Homo sapiens sur le modèle ponctuationniste. Il existe de fait, dans la préhistoire et dans l'histoire humaine, des périodes d'équilibre et de

\footnotetext{
${ }^{8}$ Gould donne l'exemple de la phylogenèse des hominidés. Homo sapiens serait le résultat d'un phénomène de spéciation qui le distingue des autres espèces, avec qui il coexiste à l'origine et qu'il remplace progressivement. L'insistance sur la coexistence (trois espèces d'hominidés, il y a seulement 40000 ans) souligne qu'une espèce, Homo sapiens, est apparue soudainement et qu'elle était capable aussi bien de coexister avec d'autres espèces que de les remplacer.
}

stabilité ponctuées par des périodes de transition, d'équilibre et de stabilité, ponctuées par des périodes d'évolution rapide. La naturalisation de la vie humaine doit-elle aller jusque-là ? L'accélération constante de l'histoire humaine permet-elle cette transposition de constatations d'origine paléontologique et préhistorique? Nous en doutons sérieusement.

Gould donne un appendice général au chapitre sur l'équilibre ponctué, en retraçant l'histoire et l'expansion de sa théorie, mais aussi le rapport conflictuel avec le créationnisme aux États-Unis. Le créationnisme est accusé de réinterpréter le ponctuationnisme, en le détournant de son sens, de l'assimiler au saltationnisme, en transposant ce que Gould nomme « l'authentique discontinuité entre espèces » dans un autre discours (p. 1381). L'évocation du conflit avec les créationnistes nous introduit aux questions d'éducation et de didactique. Gould constate la présence de l'équilibre ponctué dans les manuels à l'usage des étudiants et il reconnaît la précision de cette présentation dans ces manuels. Cet appendice donne donc diverses conséquences de la théorie des équilibres ponctués, surtout dans la société américaine, et s'appesantit un peu longuement sur les attaques dont l'auteur a été l'objet.

Les derniers chapitres du livre traitent surtout du rapport de la contrainte et de l'adaptation (Gould précise qu'il s'agit de structure et de fonction) dans l'ontogenèse et la phylogenèse. Ce sont sans doute les passages les plus originaux de l'ouvrage, dans la mesure où ils concernent les mécanismes causaux des équilibres ponctués. La réhabilitation de la contrainte structurale est intéressante si, et seulement si, elle est fondée. Peut-on réellement toucher ces déterminismes internes qui influenceraient l'histoire évolutive du développement? L'auteur donne ici des exemples (qui ne sont pas pour autant des preuves) à propos des mécanismes à l'œuvre dans les hétérochronies du développement. La néoténie chez les urodèles est l'exemple souvent invoqué par les auteurs les plus divers (cf. p. 1456), mais Gould revient surtout sur l'huître jurassique du genre Gryphæa qu'il a étudiée dans les années 1970. La question de l'hétérochronie du développement chez les spécimens de ce genre demeurait controversée tant qu'on ne pouvait pas bien connaître leur âge chronologique. Cette découverte permit de conclure : « La tendance évolutive ayant modifié simultanément la taille et la forme chez Gryphra, avait réellement résulté d'un processus de néoténie » (p. 1461). L'hétérochronie aurait été le moyen d'obtenir simultanément une série d'avantages, comme l'accroissement de la taille et de la largeur, la diminution de l'enroulement, donc la stabilité ; la néoténie aurait conservé une forme juvénile au stade adulte en maintenant une vitesse de développement importante, d'où l'accroissement mentionné. L'évolution hétérochronique de Gryphæa incurva, huitre fossile du jurassique, serait donc d'origine néoténique. L'escargot fossile Cerion uva permet, quant à lui, de montrer que la contrainte 
allométrique est une base de la variation exprimée dans l'évolution. D'où un autre aspect de la remise en cause du rôle de la sélection naturelle : les contraintes de développement seraient sources, à elles seules, de bon nombre de phénomènes évolutifs.

La reconnaissance des homologies dans de nombreuses espèces se précise à travers des structures biomoléculaires et des plans de construction homologues. La vaste étendue de ces homologies (par exemple, chez les mammifères) fait douter $d u$ " contrôle du rythme et de l'orientation du changement évolutif uniquement déterminé par la force fonctionnaliste de la sélection naturelle » (p. 1 494). Finalement, Gould convoque des découvertes de l'évo-dévo (biologie moléculaire de l'évolution et du développement) quant à l'homologie génétique supposée sous-tendre l'évolution : il parle de parallélisme génétique au sujet des gènes du développement, dont la découverte fut, de fait, une révolution scientifique majeure des vingt dernières années du $X X^{\mathrm{e}}$ siècle. Le parallélisme a été défini une première fois par le néolamarckien Edward Cope au sens où des genres qui « appartiennent à un même groupe de parallèles parcourent, durant leur ontogenèse complète, une partie plus ou moins longue d'une même voie de développement (et de phylogenèse) »; d'où l'idée que «l'adulte d'un genre donné peut être pratiquement identique à la forme juvénile d'un autre genre » (p. 1515). L'homologie suppose, quant à elle, un ancêtre commun et la réalisation du même archétype. En ce sens, sur le plan des mécanismes de développement, l'homologie donne au parallélisme son sens fondamental.

Gould se lance alors dans la justification des théories de l'archétype concernant les découvertes récentes de la génétique du développement. Selon les travaux de Theissen et Saedler ${ }^{9}$ sur les gènes $\mathrm{ABC} \mathrm{d}^{\prime}$ Arabidopsis thaliana, «la perte de fonction simultanée des gènes de classe $A B C$ conduit à la transformation de toutes les pièces florales en feuilles, corroborant la conception de Goethe selon laquelle les feuilles représentent un état de base dans le développement » (p. 1532). Ces gènes de forme commandent la formation des feuilles et des pièces florales, il y a une homologie sériée de ces organes apparemment différents. L'auteur commente ensuite le modèle de Lewis sur la différenciation morphologique chez la drosophile, commandée par les huit gènes Hox. Le modèle de Lewis permit d'établir une homologie profonde entre les divers groupes animaux, en particulier entre vertébrés et invertébrés ; il existe " une frappante similitude de l'action des gènes Hox de vertébrés [...] et de celle des gènes Hox d'insectes » (p. 1551). Cependant, les découvertes faites en évo-dévo ne confirment que partiellement les homologies vues par Geoffroy Saint-Hilaire entre les segments des arthropodes et les vertébrés. La convocation de cette théo-

\footnotetext{
9 Theissen, G., Saedler, H., 2001. Plant biology: Floral quartets,
} Nature, 409, 6819, 469-471. rie $\mathrm{du} \mathrm{XIX}^{\mathrm{e}}$ siècle a ici pour but de mettre en lumière ses révisions et ses vérifications partielles dans la génétique contemporaine du développement (homologie segmentaire et homologie par inversion dorso-ventrale du plan d'organisation). La maîtrise des gènes du développement a permis, entre autres, l'expression d'yeux surnuméraires, par exemple sur les antennes de drosophile (en faisant s'exprimer un gène régulateur $\operatorname{Pax}-6$ de la souris chez Drosophila). Gould parle alors de « conservation évolutive de la voie développementale sous-tendant l'édification des yeux d'insectes et de vertébrés ", en rapport avec la question de la convergence dans l'évolution (p. 1573). Que conclure de toutes les découvertes relatives aux gènes Hox en matière d'évolution ? En quoi l'histoire phylogénétique des gènes Hox vient-elle à l'appui de la théorie de l'évolution selon Gould ? Un ensemble initial de gènes du développement aurait déterminé l'histoire des bilatériens depuis un ancêtre commun et il y aurait eu une sorte de canalisation des directions évolutives. Certains gènes Hox peuvent avoir perdu leur fonction initiale ou avoir été réaffectés à d'autres fonctions, ils peuvent présenter des stases qu'ils conservent au cours de l'évolution. De plus, l'acquisition des spécialisations anatomiques peut s'expliquer par une restriction dans l'expression des gènes Hox. Ainsi, les « règles d'action des gènes Hox représentent des contraintes ", mais leur niveau d'expression traduirait des possibilités de flexibilité et de brusques changements (p. 1648).

\section{Conclusion}

La conclusion de Gould sur toute cette question des équilibres ponctués se fait nettement sentir dans les deux derniers chapitres. L'auteur explique que l'usage actuel d'une adaptation du vivant peut venir d'un «changement imprévu de fonction », d'une adaptation antérieure ayant «un rôle très différent» (p. 1719). C'est ce qu'il appelle une « exaptation » (p. 1723), et il s'efforce de donner des exemples probants : l'aplatissement de la tête du lézard est apparue avant l'occupation par ce dernier des fissures, et pour d'autres raisons, mais cela facilite grandement «l'ajustement [...] de l'organisme à son nouvel environnement » (p. 1727). Un autre exemple d'exaptation, moléculaire cette fois, est celui du cristallin oculaire du calmar Euprymna scolopes : Gould cite un texte de Montgomery et McFall-Ngai (1992) qui écrivent : «Il se pourrait que l'aldéhyde desydrogénase ait d'abord été recrutée à cette fin, puis qu'elle ait été secondairement convertie chez certaines espèces, afin d'accomplir un rôle essentiellement structural, comme dans la lentille du photophore » (p. 1741). C'est bien ce que Gould appelle exaptation. Pour mieux faire comprendre son raisonnement, l'auteur a recours à l'analogie de l'art : sous une coupole byzantine, il existe quatre espaces triangulaires, 
les pendentifs, qui «se forment en tant que conséquences secondaires, structuralement nécessaires, du projet fondamental de l'architecte de monter une coupole sous un ensemble de 4 voûtes arrondies, disposées en carré » (p. 1747). Bien que conséquentes, ces structures sont utiles. De même, les structures des organismes biologiques appellent des expansions structurales, des exaptations à partir de la formation primaire de l'organisme, le point commun entre ces deux ordres de choses (l'art et la biologie) étant le fait que « les organisations géométriques tridimensionnelles les plus simples et les plus universelles doivent nécessairement engendrer des séries entières d'expansions structurales... » (p. 1760). Un exemple biologique est celui de l'escargot, qui peut laisser un espace cylindrique vide dans l'axe de la coquille, autour duquel il s'enroule; cet espace est utilisable par certaines espèces pour y faire incuber leurs œufs. Un exemple paléontologique est celui de l'élan irlandais, Megaloceros giganteus, dont les apophyses vertébrales, très grandes au niveau de l'épaule, furent des conséquences destinées à équilibrer par la musculature le poids hors norme de la ramure (35 kg). L'omniprésence des expansions structurales souligne une hiérarchie dans l'évolution : des conséquences $\mathrm{d}^{\prime}$ une structure anatomique et morphologique tiennent lieu d'adaptations secondaires relevant d'une adaptation primaire (par exemple, le développement de la ramure dans le cas de Megaloceros giganteus). À partir de là, Gould voit l'existence d'une réserve de potentialités exaptatives, par exemple au niveau des gènes Hox, dont la flexibilité des régulations est à même d'engendrer de nouvelles adaptations, c'est-à-dire, finalement, de nouvelles potentialités. Les expansions structurales de Gould constituent des contraintes de structure, sources d'éventuelles adaptations sans être des adaptations directes.

Enfin, et c'est la conclusion logique de cet ouvrage, le lecteur assiste à une remise en cause en bonne et due forme de la façon dont Darwin et les néodarwiniens extrapolent une évolution graduelle à partir d'archives géologiques ponctuées, en invoquant l'imperfection de ces archives. Darwin aurait ramené, «dans le cadre interprétatif de l'extrapolationnisme, ce qui semble se présenter comme des extinctions de masse» (p. 1817). L'imperfection du matériel dont disposent les géologues et les paléontologues renverrait à un étalement de "l'apparente simultanéité » des extinctions de masse "sur une période suffisamment longue» (p. 1819). La remise en cause de ce présupposé uniformitariste appelle les hypothèses contemporaines de l'extinction massive des espèces à la fin de l'ère secondaire, en rapport, entre autres, avec la présence massive d'iridium, détectée à la frontière du Crétacé et du tertiaire et provenant peut-être d'un gros météorite. L'hypothèse catastrophiste retrouve de ce fait une certaine aura, d'où la relecture du gradualisme admis autrefois dans la disparition des dinosaures au profit d'une « disparition véritablement catastrophique » (p. 1831).

Comme conclusion plus personnelle, nous voudrions dire que la théorie ponctualiste ne se présente pas comme une théorie auxiliaire de l'évolution (comme la théorie neutraliste ou la théorie endosymbiotique, qui ne remettent pas en cause le néodarwinisme dans son ensemble, mais le complètent sur des terrains où il ne peut pas assumer un rôle prédictif). Elle constitue une nouvelle théorie de l'évolution à part entière, théorie à l'intérieur de laquelle on serait amené à faire des prédictions (poppériennes) correspondant avec les faits livrés surtout par la paléontologie. La théorie des équilibres ponctués s'avère à même de supplanter très largement la théorie néodarwinienne. L'enthousiasme de Stephen Jay Gould est néanmoins à modérer par la résistance du néodarwinisme, sous l'angle d'autres disciplines biologiques. Enfin, ce livre constitue une somme qui fera date, non seulement en biologie générale et en paléontologie, mais aussi en histoire et philosophie des sciences. 